\title{
Dynamic Shapley Value in the Game with Perishable Goods*
}

\author{
Yin $\mathbf{L i}^{12}$, Ovanes Petrosian ${ }^{1}$ and Jinying Zou $^{1}$ \\ 1 St. Petersburg State University, \\ 7/9 Universitetskaya nab., Saint Petersburg 199034, Russia, \\ 2 School of Economics and Management, Yanan University, Yan'an, 716000, China \\ E-mail: liyinrus@outlook.com, petrosian.ovanes@yandex.ru \\ jesco4482@gmail.com
}

\begin{abstract}
The paper investigates two-stage stochastic minimum spanning tree games with perishable goods. The cooperative behaviour of the players is defined. At each stage, all players jointly take action to construct a network with a cost matrix. At the second stage, a particular player may leave the game, and the probability of this leaving depends on the cooperative behaviour of all players at the first stage. At each stage game, the total cost of the spanning tree is calculated to include the sum of the costs of the contained edges and the cost of the loss of perishable goods expended on that edge of the spanning tree. The characteristic functions in the game are considered, and the dynamic Shapley values are modified. The time consistency of the dynamic Shapley values is studied.
\end{abstract}

Keywords: dynamic games, minimum cost spanning tree game, stochastic games.

\section{Introduction}

This article studies two-stage stochastic minimum cost spanning tree games with perishable goods. Perishable goods are those products whose quality or value decreases over time, such as vegetables, flowers or seafood. Due to differences in the timing of transport and storage methods, perishable goods are more complex in terms of cost loss and risk to players as they are transported through the logistics network. In China, the loss of perishable food products has been calculated to be between $20 \%$ and $30 \%$ of the production costs of the companies involved (Wan and Cao, 2019). Research on the loss of perishable goods can be traced back to the 1960s and has been systematically reviewed by Nahmias and Janssen et al. in the field of inventory control of perishable goods (Nahmias, 1982; Janssen, 2016). The minimum cost spanning tree game is a classical model of cooperative games derived from the minimum cost spanning tree problem and was formally proposed by Claus and Kleitman (Claus, 1973) in 1973. The most famous solution to the minimum spanning tree game is the Bird allocation proposed by Bird (Bird, 1976). Dutta and Kar (Dutta, 2004) proposed the Dutta-Kar allocation, which satisfies cost monotonicity and is in the core. The literature (Bird, 1976) also introduced the concept of an irreducible core. Another important solution is called the Folk allocation (Bergantiños and Vidal-Puga, 2007) which is the Shapley value of the game corresponding to the irreducible graph.

In dynamic cooperative game theory, the time consistency of the solution is a crucial concern. That is, players should be able to make an agreement at the beginning of the game so that at the end of the game, they receive the gains specified

${ }^{*}$ This work was supported by the China Scholarship Council (No.201508090030).

https://doi.org/10.21638/11701/spbu31.2021.20 
in the solution, as previously agreed. In the case of time inconsistency, the players in the game may break the initial agreement. In the literature $(\mathrm{Li}, 2016)$, the case in which a minimum spanning tree game was combined with a stochastic game was considered for the first time. It considered the construction of dynamic Shapley values in a two-stage minimum cost spanning tree game. At each stage, when the players take different strategies, the costs of the edges constructed between the players in the network may be different, and thus the associated minimum cost spanning tree may be different, and the cost-sharing solutions obtained will be different. It is assumed in the research that all players in the game adopt cooperative behaviour in order to minimise the expected sum of their costs. The characteristic function is defined. The dynamic Shapley value in the game is constructed, and the problem of time consistency of the solution is considered. Further, in the literature (Li, 2017), a two-stage stochastic minimum cost spanning tree game with perishable goods is discussed.

In the goods transportation model, the value of a good varies following its utility as the shipping time increases; see Fig. 1. Three cases are possible:

- The blue curve shows that the good's utility remains the same with an increase in the shipping time (umbrellas, plastic toys for children, etc.).

- The green curve shows that the good's utility grows with an increase in the shipping time (wine, antiques, etc.).

- The red curve shows that that the good's utility drops with an increase in the shipping time. This applies to perishable goods, i.e., the ones becoming cheaper over time (flowers, fruits, etc.).

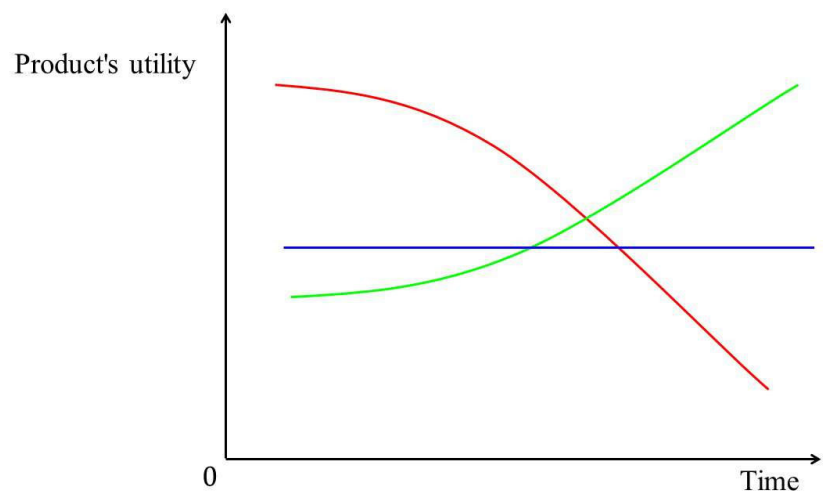

Fig. 1. Three types of goods with different utility dynamics.

The perishable goods can be divided into two groups:

1. The goods with a fixed expiration date, without any value loss on a certain period of time;

2. The continuously perishing goods, with a value decreasing proportionally to the shipping time.

The second group of perishable goods is studied in this paper. 
This article follows the research $(\mathrm{Li}, 2017)$. At each stage of the game, the players act together to construct a network, and a cost matrix of the network is obtained. The network is built on a set of sources and vertices which represent the players. In the research $(\mathrm{Li}, 2016)$, the minimum cost spanning tree is obtained after defining this network with the cost matrix, using algorithms based on classical graph theory (Dijkstra, 1959; Kruskal, 1956; Prim, 1957). In contrast, in the minimum cost spanning tree games with perishable goods, the total cost of the spanning tree is calculated to include the sum of the costs of the contained edges and the cost of the loss of perishable goods expended on that edge of the spanning tree. It means that the loss of perishable goods is generally less important for the same player on two different spanning trees when the vertex of the player is closer to the source. After the first stage, a particular player leaves the game with a probability that depends on the cooperative behaviour of all players in the first stage. The total cost to the players in this two-stage game is the sum of their costs in the first and second stages. All players select cooperative actions. We consider the strategy profiles of players with the minimum expected total cost of two-stage game as the cooperative strategy. The characteristic functions in the game are considered, and the dynamic Shapley values are modified. The time consistency of the dynamic Shapley values is studied.

\section{The Model}

First of all, we describe the game model. When defining the two-stage game, we begin with the one-stage game, which is a spanning tree game. Following (Li, 2016), introduce the main definitions and necessary background from graph theory.

Assume that $n$ players are in $n$ different positions. To obtain perishable goods, the players directly or indirectly connect to the source. After the first stage, player $m \in N$ can leave the game with a probability $p$ depending on the previous behavior of players. If player $m$ leaves the game, the set of players changes. Otherwise (player $m$ stays in the game), the game is repeated by analogy at the second stage. The diagram of this game is presented in Fig. 2.

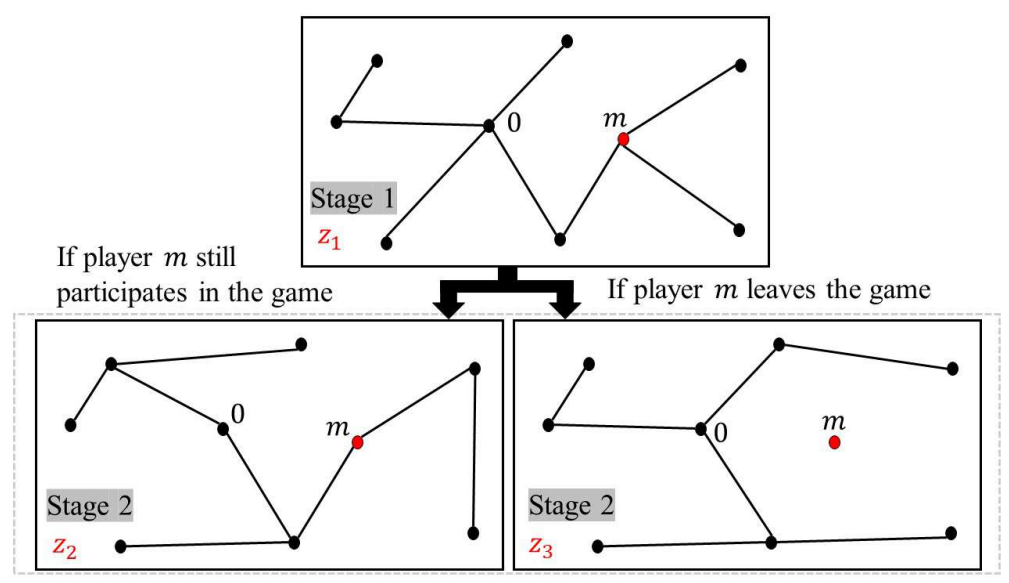

Fig. 2. The diagram of the game. 
Definition 1. Let $N=\{1, \ldots, n\}$ be a finite set of players, $\{0\}$ be the source, and $N^{\prime}=N \cup\{0\}$.

Definition 2. A graph defined on the set $N^{\prime}$ will be denoted by $G\left(N^{\prime}, E\right)$, where $E=\left\{(i, j):(i, j) \in L \subset N^{\prime}\right\}$.

Definition 3. A pair $(i, j)$ is called an edge in a graph $G\left(N^{\prime}, E\right)$ if $(i, j) \in E$, $\forall i, j \in N$.

Definition 4. Two vertices $i$ and $j \in N^{\prime}$ are said to be connected in a graph $G\left(N^{\prime}, E\right)$ if $\exists\left(i_{1}, i_{2}\right),\left(i_{2}, i_{3}\right), \ldots,\left(i_{n-1}, i_{n}\right):\left(i_{k}, i_{k+1}\right) \in G\left(N^{\prime}, E\right), 1 \leq k \leq n-1$, $i_{1}=i$, and $i_{n}=j$.

Definition 5. A graph $G\left(N^{\prime}, E\right)$ is said to be connected on the set $N^{\prime}$ if any two vertices $i, j \in N^{\prime}$ are connected in $G\left(N^{\prime}, E\right)$.

Definition 6. The set of all connected graphs on the set $N^{\prime}$ will be denoted by $\mathcal{G}_{N^{\prime}}$.

Definition 7. Associate with any $i$ and $j$ in a graph $G\left(N^{\prime}, E\right)$ cost $c_{i j}$ on the edge $(i, j), i \neq j \in N^{\prime}$, where $c_{i j}=c_{j i} \in \mathbb{R}^{+}$and $\forall i \in N^{\prime}: c_{i i}=+\infty$. The cost $c_{i 0}=c_{0 i}$ on the edge $(i, 0), i \in N$, is a nonnegative constant.

Depending on the interpretation of the model, the cost on an edge $(i, j)$ can be the consumption of some resource (e.g., fuel consumption when shipping a cargo from vertex $i$ to vertex $j$ ) or the value of some service (e.g., the price of connecting vertex $i$ to vertex $j$ ).

The cost on a graph $G\left(N^{\prime}, E\right)$ can be represented as a cost matrix

$$
C=\left\{c_{i j}\right\}_{(n+1) \times(n+1)} .
$$

Note that a cost matrix is nonnegative and symmetric and has order $(n+1) \times$ $(n+1)$.

Example 1. Consider an example shown in Fig. 3. The red vertex 0 is the source, and the four vertices indicated by green numbers are players. In this example, the graph is complete, and each edge has a specific cost.

The corresponding cost matrix is given by

$$
C=\left\{c_{i j}\right\}_{5 \times 5}=\begin{gathered}
0 \\
1 \\
2 \\
3 \\
4
\end{gathered}\left(\begin{array}{ccccc}
0 & 1 & 2 & 3 & 4 \\
6 & 6 & 3 & 15 & 11 \\
3 & 8 & 8 & 5 & 10 \\
15 & 5 & 4 & 4 & 12 \\
11 & 10 & 12 & 9 & 9 \\
+\infty
\end{array}\right), \forall i, j \in\{0,1,2,3,4\}
$$

\subsection{Stage Game with Spanning Tree}

Definition 8. (Li, 2016) At each stage, player $i$ chooses a strategy vector $(\mathrm{Li}, 2016)$

$$
x_{i}=\left(x_{i, 1}, \ldots, x_{i, i-1}, x_{i, i+1}, \ldots, x_{i, n}\right),
$$

where $x_{i, j} \in X_{i, j}$ denotes an action of player $i$ against player $j, \forall i, j \in N$. Similarly, $x_{j, i} \in X_{j, i}$ is an action of player $j$ against player $i$. 


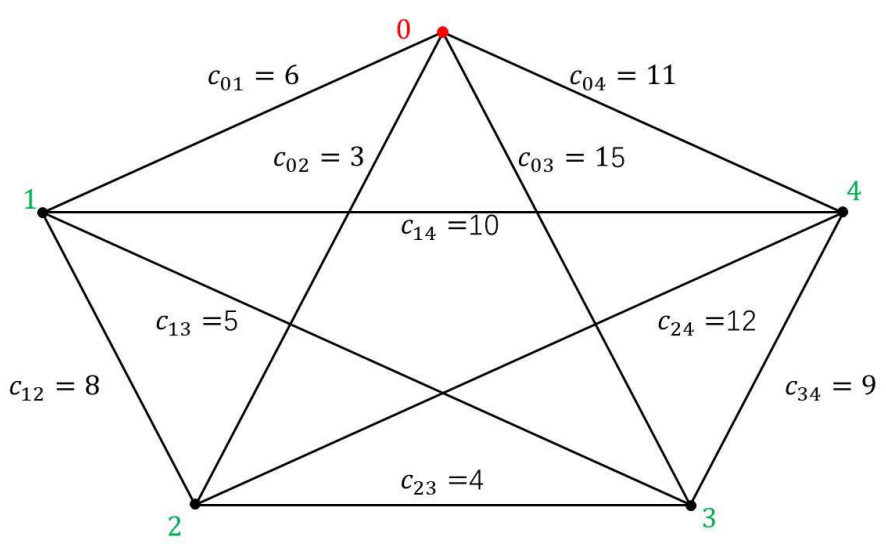

Fig. 3. Cost on complete graph with four players.

Definition 9. (Li, 2016) The cost on an edge $(i, j)$ of a graph $G\left(N^{\prime}, E\right)$ is defined in the following way:

$$
c_{i j}=c_{j i}=f_{c}\left(x_{i, j}, x_{j, i}\right), c_{i 0}=c_{0 i}>0, \forall i, j \in N .
$$

where a function $f_{c}$ maps the set of all strategies of players $i, j$ into the set $\mathbb{R}^{+} \cup$ $\{+\infty\}$ of all admissible costs on the edges $(i, j) \in E$.

In other words, the cost on an edge $c_{i j}=c_{j i}$ is completely defined by the strategies $x_{i, j}, x_{j, i}$ of players $i, j$, where $x_{i, j} \in X_{i, j}, x_{j, i} \in X_{j, i}, \forall i, j \in N$. The cost on all edges between the players is defined by a given strategy profile $x=$ $\left(x_{1}, \ldots, x_{n}\right)$. Note that $C_{x}=\left\{c_{i j}\right\}_{(n+1) \times(n+1)}$ is a unique cost matrix constructed by Definition 9 . Thus, for a cost matrix $C_{x}=\left\{c_{i j}\right\}_{(n+1) \times(n+1)}$, there is a unique graph $G\left(N^{\prime}, E\right) \in \mathcal{G}_{N^{\prime}}$ on the set $N^{\prime}$. Here $\mathcal{G}_{N^{\prime}}$ denotes the set of all possible connected graphs on the set $N^{\prime}$.
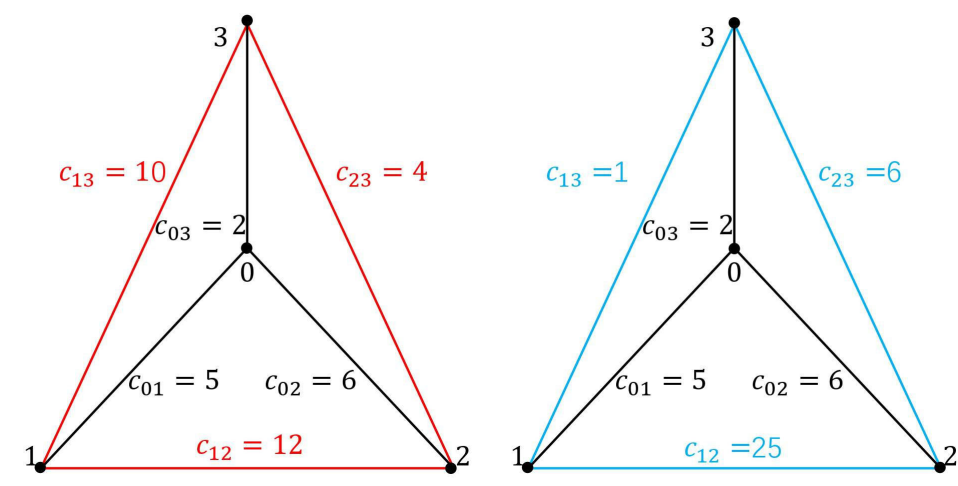

Fig. 4. Graphs with different costs on edges corresponding to different strategies of players. 
Example 2. Consider a three-player game. Let the function $f_{c}$ be $f_{c}=x_{i, j} \times x_{j, i}$, where $x_{i, j} \in X_{i, j}, x_{j, i} \in X_{j, i}$. If players 1-3 choose the strategy profiles

$$
x_{1}^{\prime}=\left(x_{1,2}^{\prime}, x_{1,3}^{\prime}\right)=(3,10), x_{2}^{\prime}=\left(x_{2,1}^{\prime}, x_{2,3}^{\prime}\right)=(4,2), x_{3}^{\prime}=\left(x_{3,1}^{\prime}, x_{3,2}^{\prime}\right)=(1,2),
$$

respectively, we easily calculate the cost on all edges between the players. In the lefthand part of Fig. 4, the red edges are obtained under the strategy profile $\left(x_{1}^{\prime}, x_{2}^{\prime}, x_{3}^{\prime}\right)$. For example, for the edge $(1,2)$, we have $c_{12}=c_{21}=x_{1,2}^{\prime} \times x_{2,1}^{\prime}=3 \times 4=12$.

When choosing the strategy profiles

$$
x_{1}^{\prime \prime}=\left(x_{1,2}^{\prime \prime}, x_{1,3}^{\prime \prime}\right)=(5,1), x_{2}^{\prime \prime}=\left(x_{2,1}^{\prime \prime}, x_{2,3}^{\prime \prime}\right)=(5,2), x_{3}^{\prime \prime}=\left(x_{3,1}^{\prime \prime}, x_{3,2}^{\prime \prime}\right)=(1,3),
$$

the costs on all edges between the players varies. In the right-hand part of Fig. 4, the blue edges are obtained under strategy profile $\left(x_{1}^{\prime \prime}, x_{2}^{\prime \prime}, x_{3}^{\prime \prime}\right)$. For example, for the edge $(2,3)$, we have $c_{23}=c_{32}=x_{2,3}^{\prime \prime} \times x_{3,2}^{\prime \prime}=2 \times 3=6$.

Definition 10. ( $\mathrm{Li}, 2016)$ A minimum cost spanning tree on the set $N^{\prime}$ is the tree

$$
T\left(N^{\prime}, C_{x}\right)=\arg \min _{G \in \mathcal{G}_{N^{\prime}}} \sum_{(i, j) \in G\left(N^{\prime}, E\right)} c_{i j},
$$

where $C_{x}=\left\{c_{i j}\right\}_{(n+1) \times(n+1)}$ denotes a cost matrix on a graph $G\left(N^{\prime}, E\right)$, which is uniquely defined by the strategy profile $x=\left(x_{1}, \ldots, x_{n}\right)$. Here $\mathcal{G}_{N^{\prime}}$ denotes the set of all possible connected graphs on the set $N^{\prime}$.

Definition 11. (Li, 2016) The total cost on the edges of a minimum cost spanning tree $T\left(N^{\prime}, C_{x}\right)$ is given by

$$
C\left[T\left(N^{\prime}, C_{x}\right)\right]=\sum_{(i, j) \in T\left(N^{\prime}, C_{x}\right)} c_{i j}
$$

Obviously, a graph with a minimum cost represents a tree. Otherwise, we would eliminate an extra edge to get a connected graph with a smaller cost.

Example 3. As an illustrative example, consider a one-stage three-player game. Let $N=\{1,2,3\}$ be the set of players, $\{0\}$ be the source, and $N^{\prime}=N \cup\{0\}$. The corresponding graph on the set $N^{\prime}$ is shown in Fig. 5. Assume that edges $(0,1),(0,2)$, and $(0,3)$ are fixed, and the cost on these edges is $c_{01}=c_{10}=30, c_{02}=c_{20}=51$, and $c_{03}=c_{30}=16$, respectively. The function $c_{i j}=f_{c}\left(x_{i, j}, x_{j, i}\right)$ has the form $f_{c}\left(x_{i, j}, x_{j, i}\right)=x_{i, j} \times x_{j, i}, \forall i, j \in N$.

All possible costs on the edges $(i, j), \forall i \neq j \in\{1,2,3\}$, are combined in Table 1 . Here the red, green, and blue colors indicate different edges. Let $X_{2,3}=\{2,21\}$ be the set of actions of player 2 against player 3 , and $X_{3,2}=\{12,19\}$ be the set of actions of player 3 against player 2, where $x_{2,3} \in X_{2,3}$ and $x_{3,2} \in X_{3,2}$. If player 1 chooses the action $x_{2,3}=21$, and player 2 chooses the action $x_{3,2}=12$, then the cost makes up $c_{23}=f_{c}\left(x_{2,3}, x_{3,2}\right)=21 \times 12=252$.

Different strategies result in different cost matrices, and a minimum spanning tree can be obtained for each cost matrix. Assume that the players choose their strategies, and the strategy profile in the game is

$$
x_{1}=\left(x_{1,2}, x_{1,3}\right)=(6,3), x_{2}=\left(x_{2,1}, x_{2,3}\right)=(7,2), x_{3}=\left(x_{3,1}, x_{3,2}\right)=(9,12) .
$$


Table 1. All possible costs on edges under different actions of players

\begin{tabular}{|c|c|c|c|c|c|c|c|c|c|c|c|}
\hline \multirow{2}{*}{\multicolumn{2}{|c|}{$c_{12}$}} & \multicolumn{2}{|c|}{$X_{2,1}$} & \multirow{2}{*}{\multicolumn{2}{|c|}{$c_{13}$}} & \multicolumn{2}{|c|}{$X_{3,1}$} & \multirow{2}{*}{\multicolumn{2}{|c|}{$c_{23}$}} & \multicolumn{2}{|c|}{$X_{3,2}$} \\
\hline & & 7 & 10 & & & 9 & 11 & & & 12 & 19 \\
\hline \multirow{2}{*}{$X_{1,2}$} & 6 & 42 & 60 & & 3 & 27 & 33 & \multirow{2}{*}{$X_{2,3}$} & 2 & 24 & 39 \\
\hline & 17 & 119 & 170 & & 15 & 135 & 165 & & 21 & 252 & 399 \\
\hline
\end{tabular}

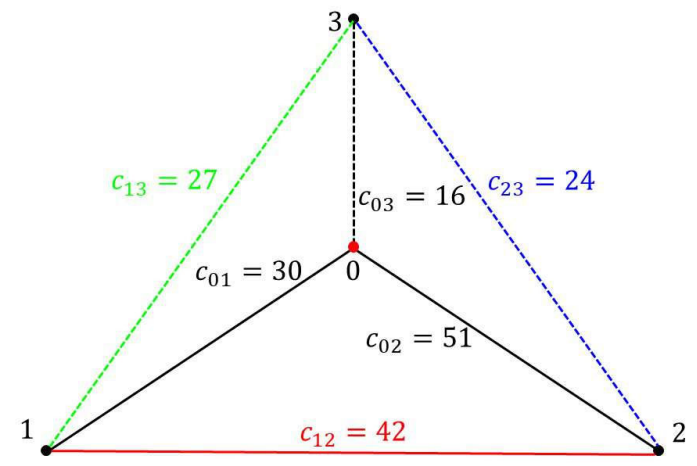

Fig. 5. Minimum cost spanning tree for graph constructed by players.

The graph with cost corresponding to the strategy profile $(9,12)$ is shown in Fig. 5.

The cost matrix takes the form

$$
C_{x}=\begin{gathered}
0 \\
1 \\
2 \\
3
\end{gathered}\left(\begin{array}{cccc}
\infty & 1 & 2 & 3 \\
30 & +\infty & 51 & 16 \\
51 & 42 & +\infty & 27 \\
16 & 27 & 24 & +\infty
\end{array}\right)
$$

After obtaining the cost matrix, the minimum cost spanning tree $T\left(N^{\prime}, C_{x}\right)$ on the graph consists of edges $(0,3),(1,3)$, and $(2,3)$; see dotted lines in Fig. 5 . Therefore, the total cost in the minimum cost spanning tree $T\left(N^{\prime}, C_{x}\right)$ under the strategy profile $x=\left(x_{1}, x_{2}, x_{3}\right)$ is

$$
C\left[T\left(N^{\prime}, C_{x}\right)\right]=67,
$$

where $x_{1}=\left(x_{1,2}, x_{1,3}\right)=(6,3), x_{2}=\left(x_{2,1}, x_{2,3}\right)=(7,2), x_{3}=\left(x_{3,1}, x_{3,2}\right)=(9,12)$.

Definition 12. A route in a tree $T\left(N^{\prime}, C_{x}\right)$ is a sequence of vertices $i_{1}, i_{2}, \ldots, i_{K}$ and edges $\left(i_{k}, i_{k+1}\right)$ for all $k \in[1, K-1]$ and $K \in \mathbb{N}^{+}, K \geq 2$. We say that this route passes the edges $\left(i_{k}, i_{k+1}\right)$ and the vertices $i_{1}, i_{2}, \ldots, i_{K}$. If a route $i_{1}, i_{2}, \ldots, i_{K}$ in a tree $T\left(N^{\prime}, C_{x}\right)$ passes none of the edges $\left(i_{k}, i_{k+1}\right)$ twice, it is called a path. In this case, vertex $i_{1}$ is called the start vertex of the path, and vertex $i_{K}$ the end vertex of the path. In a tree $T\left(N^{\prime}, C_{x}\right)$, we denote by $P_{i_{1} i_{K}}$ a path from vertex $i_{1}$ to vertex $i_{K}$, and by $P_{m 0}$ a path from any vertex $m \in N$ to the source $\{0\}$. 
Definition 13. Vertex $j$ is called a predecessor of vertex $m$ if $P_{j 0} \varsubsetneqq P_{m 0}$, where $P_{j 0}$ is a path from source $\{0\}$ to vertex $j$ in a tree $T\left(N^{\prime}, C_{x}\right), j \in N$.

Definition 14. Consider a tree $T\left(N^{\prime}, C_{x}\right)$. Assume that for any vertex $m \in N$, a path $P_{m 0}$ from the start vertex $m$ to the end vertex $\{0\}$ passes vertices $m_{1}$, $m_{2}, \ldots, m_{K}$ and edges $\left(m_{k}, m_{k+1}\right)$, where $k \in[1, K-1], m_{1}=m, m_{K}=\{0\}$, $K \in \mathbb{N}^{+}$, and $K \geq 2$. Then vertex $m_{k}$ is called a direct predecessor of vertex $m_{k+1}$. A direct predecessor of vertex $m$ will be denoted by $P(m)$. If the path includes only two vertices $m$ and $m^{\prime}$, then $P(m)=m^{\prime}$.

Definition 15. For any subtree $T\left(S, C_{x}^{S}\right)$ of a tree $T\left(N^{\prime}, C_{x}\right), S \varsubsetneqq N$, consider vertex $i \in S$. If after deleting this vertex $i$ in the subtree $T\left(S, C_{x}^{S}\right)$, for all $j \in S \backslash\{i\}$ the path $P_{j 0}$ does not exist, then the set of all edges of the subtree $T\left(S, C_{x}^{S}\right)$ is called a branch in the tree $T\left(N^{\prime}, C_{x}\right)$. This set will be denoted by $B^{i}$.

Definition 16. Vertex $i \in N$ is said to be terminal in a tree $T\left(N^{\prime}, C_{x}\right)$ if there does not exist a vertex $j \in N \backslash\{i\}$ such that $P_{0 i} \subset P_{0 j}$ and $P_{0 j} \in T\left(N^{\prime}, C_{x}\right)$.

Example 4. Consider an example in Fig. 6. Here $\{0\}$ is the source in a spanning tree $T\left(N^{\prime}, C_{x}\right)$. The following can be established directly by definition.

- All blue edges $\left(0, j_{1}\right),\left(j_{1}, j_{2}\right),\left(j_{2}, j_{3}\right)$ make a path $P_{m 0}$ from source $\{0\}$ to vertex $m$.

- Each of the vertices $j_{1}, j_{2}, j_{3}$ is a predecessor of vertex $m$. Moreover, vertex $j_{3}$ is a direct predecessor of vertex $m$, i.e., $P(m)=j_{3}$.

- Let $S$ be the set of all red vertices, and $m \in S$. Then $B^{m}$ is the set of all edges in the yellow domain.

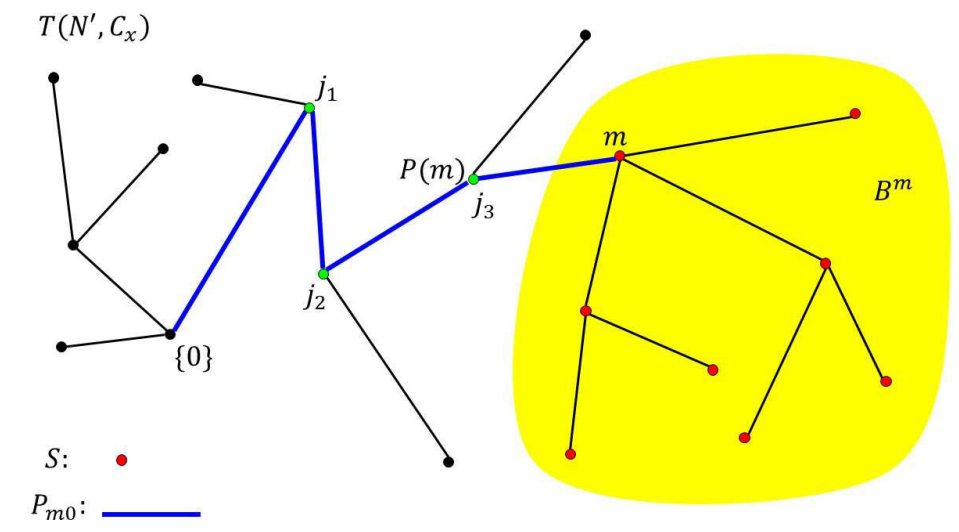

Fig. 6. Path $P_{m 0}$, direct predecessor of vertex $m$, predecessor of vertex $m$, and branch $B^{m}$.

Definition 17. (Li, 2016) The probability that player $m$ will leave the game at the second stage is calculated as

$$
p=\frac{\sum_{(i, j) \in B^{m}} c_{i j}}{C\left[T\left(N^{\prime}, C_{x}\right)\right]}
$$


where the minimum cost spanning tree $T\left(N^{\prime}, C_{x}\right)$ is given by Definition 10 on the graph $G\left(N^{\prime}, E\right)$ constructed under the strategy profile $x=\left(x_{1}, \ldots, x_{n}\right)$. Due to 15 , $B^{m}$ is a branch in $T\left(N^{\prime}, C_{x}\right)$.

\subsection{Minimum Cost Spanning Tree Game with Perishable Goods}

Definition 18. The loss in value for the perishable goods of player $i$ is given by

$$
\begin{gathered}
\mathrm{LV}=q_{i}\left(1-e^{\frac{\theta_{i}}{\sum c_{k l}}}\right), \forall i \in N,(k, l) \in P_{i 0}, \\
q_{i} \in(0,+\infty), \theta_{i} \in(0,+\infty)
\end{gathered}
$$

where $P_{i 0}$ denotes the path from source $\{0\}$ to vertex $i$, and $q_{i}$ is the cost of perishable goods of player $i$. In addition, $\theta_{i}$ is the loss parameter of player $i$ due to perishable goods, $\forall i \in N$.

According to formula (3), the longer the path $P_{i 0}$ from vertex $i$ to source $\{0\}$ is, the greater the level of LV for player $i$ will be.

Different from Definition 10, the definition of a minimum spanning tree with perishable goods is given below.

Definition 19. (Li, 2017) A minimum cost spanning tree with perishable goods on the set $N^{\prime}$ is the tree

$$
T^{\theta}\left(N^{\prime}, C_{x}\right)=\arg \min _{G \in \mathcal{G}_{N^{\prime}}}\left\{\sum_{(i, j) \in G\left(N^{\prime}, E\right)} c_{i j}+\sum_{(k, l) \in P_{i 0},(k, l) \in G\left(N^{\prime}, E\right), i \in N} q_{i}\left(1-e^{-\frac{\theta_{i}}{\sum c_{k l}}}\right)\right\},
$$

where $C_{x}=\left\{c_{i j}\right\}_{(n+1) \times(n+1)}$ denotes a cost matrix on a graph $G\left(N^{\prime}, E\right)$, and the cost matrix $C_{x}$ itself is determined using a strategy profile $x=\left(x_{1}, \ldots, x_{n}\right)$.

Definition 20. (Li, 2017) The total cost on the edges of a minimum cost spanning tree with perishable goods $T^{\theta}\left(N^{\prime}, C_{x}\right)$ is given by

$$
C\left[T^{\theta}\left(N^{\prime}, C_{x}\right)\right]=\sum_{(i, j) \in T^{\theta}\left(N^{\prime}, C_{x}\right)} c_{i j}+\sum_{(k, l) \in P_{i 0},(k, l) \in G\left(N^{\prime}, E\right), i \in N} q_{i}\left(1-e^{-\frac{\theta_{i}}{\sum c_{k l}}}\right),
$$

where $x=\left(x_{1}, \ldots, x_{n}\right)$ denotes the strategy profile of all players.

If player $m$ leaves the game, the set of players will be $N \backslash\{m\}$, and $N^{\prime} \backslash\{m\}=$ $N \backslash\{m\} \cup\{0\}$.

Definition 21. (Li, 2017) A minimum cost spanning tree with perishable goods on the set $N^{\prime} \backslash\{m\}$ is the tree

$$
\begin{gathered}
T^{\theta}\left(N^{\prime} \backslash\{m\}, C_{x \backslash\{m\}}\right)=\arg \min _{G \in \mathcal{G}_{N^{\prime} \backslash\{m\}}}\left\{\sum_{(i, j) \in G\left(N^{\prime} \backslash\{m\}, E\right)} c_{i j}\right. \\
\left.+\sum_{i \in N \backslash\{m\},(k, l) \in P_{i 0},(k, l) \in G\left(N^{\prime} \backslash\{m\}, E\right)} q_{i}\left(1-e^{-\frac{\theta_{i}}{\sum c_{k l}}}\right)\right\},
\end{gathered}
$$

where $x \backslash\{m\}=\left(x_{1}, \ldots, x_{m-1}, x_{m+1}, \ldots, x_{n}\right)$ denotes the strategy profile of all players, except the player $m$. 
Definition 22. ( $\mathrm{Li}, 2017)$ The total cost on the edges of a minimum cost spanning tree with perishable goods $T^{\theta}\left(N^{\prime} \backslash\{m\}, C_{x \backslash\{m\}}\right)$ is given by

$$
\begin{gathered}
C\left[T^{\theta}\left(N^{\prime} \backslash\{m\}, C_{x \backslash\{m\}}\right)\right]=\sum_{(i, j) \in T^{\theta}\left(N^{\prime} \backslash\{m\}, C_{x \backslash\{m\}}\right)} c_{i j} \\
+\sum_{i \in N \backslash\{m\},(k, l) \in P_{i 0},(k, l) \in T^{\theta}\left(N^{\prime} \backslash\{m\}, C_{x \backslash\{m\}}\right)} q_{i}\left(1-e^{\left.-\frac{\theta_{i}}{\sum c_{k l}}\right)}\right.
\end{gathered}
$$

where $x \backslash\{m\}=\left(x_{1}, \ldots, x_{m-1}, x_{m+1}, \ldots, x_{n}\right)$ denotes the strategy profile of all players, except the player $m$.

The value $p$ (the probability that player $m$ will leave the game) is given by Definition 17.

\section{Description of the Game}

Stage 1: At the first stage, the spanning tree game with perishable goods represents a simultaneous $n$-player game; see Fig. 2 . Therefore, at the first stage in vertex $z_{1}$, the players simultaneously choose their strategies in the form of $n$-dimensional profiles

$$
\begin{aligned}
& x^{1}=\left(x_{1}^{1}, \ldots, x_{n}^{1}\right), \\
& x_{i}^{1}=\left(x_{i, 1}^{1}, \ldots, x_{i, i-1}^{1}, x_{i, i+1}^{1}, \ldots, x_{i, n}^{1}\right), \\
& \forall i \neq j \in N,
\end{aligned}
$$

where $x_{i, j}^{1} \in X_{i, j}^{1}$ denotes an action of player $i$ against player $j$.

Stage 2: If player $m$ still participates in the game after the first stage in vertex $z_{1}$, the spanning tree game with perishable goods evolves to the second stage in vertex $z_{2}$; see Fig. 2. All players again simultaneously choose their strategies in the form of the $n$-dimensional profiles

$$
\begin{aligned}
& x^{2}=\left(x_{1}^{2}, \ldots, x_{n}^{2}\right), \\
& x_{i}^{2}=\left(x_{i, 1}^{2}, \ldots, x_{i, i-1}^{2}, x_{i, i+1}^{2}, \ldots, x_{i, n}^{2}\right), \\
& \forall i \neq j \in N,
\end{aligned}
$$

where $x_{i, j}^{2} \in X_{i, j}^{2}$ denotes an action of player $i$ against player $j$.

Then the cost matrix $C_{x^{2}}=\left\{c_{i j}\right\}_{(n+1) \times(n+1)}, \forall i \neq j \in N$ is formed on the graph $G\left(N^{\prime}, E\right)$ by the strategy profiles (7); see Definition 9 .

If player $m$ leaves the game, the set of players will be $N \backslash\{m\}$. Then the spanning tree game with perishable goods evolves to the second stage in vertex $z_{3}$; see Fig. 2 . All players, except for player $m$, simultaneously choose their strategies in the form of the $(n-1)$-dimensional profiles

$$
\begin{aligned}
x^{2} \backslash\{m\} & =\left(x_{1}^{2}, \ldots, x_{m-1}^{2}, x_{m+1}^{2}, \ldots, x_{n}^{2}\right), \\
x_{i}^{2} & =\left(x_{i, 1}^{2}, \ldots, x_{i, i-1}^{2}, x_{i, i+1}^{2}, \ldots, x_{i, n}^{2}\right), \\
\forall i & \neq j \in N \backslash\{m\},
\end{aligned}
$$

where $x_{i, j}^{2} \in X_{i, j}^{2}$ denotes an action of player $i$ against player $j$.

Then the cost matrix $C_{x^{2} \backslash\{m\}}=\left\{c_{i j}\right\}_{n \times n}, \forall i \neq j \in N \backslash\{m\}$ is formed on the graph $G\left(N^{\prime} \backslash\{m\}, E\right)$ by the strategy profiles (3.); see Definition 9. 


\section{Cooperative Game}

Assume that in the two-stage game, the cost on the edges between the players is equal to the sum of corresponding costs at the first and second stages. Consider a cooperative version of the game in which all players jointly choose their strategies to minimize the expected total cost on edges.

Define a characteristic function for the coalition $N$. Suppose that some path $z_{1}, z_{2}$ is realized during the game with perishable goods.

Thus, $x_{i}\left(z_{1}\right)=x_{i}^{1}$ and $x_{i}\left(z_{2}\right)=x_{i}^{2}, i \in N$.

Definition 23. The characteristic function for the coalition $N$ has the form

$$
\begin{aligned}
& V^{1}\left(N^{\prime}\right) \\
& =\min _{x(\cdot)}\left\{C\left[T^{\theta}\left(N^{\prime}, C_{x^{1}}\right)\right]+\left[p C\left[T^{\theta}\left(N^{\prime} \backslash\{m\}, C_{x^{2} \backslash\{m\}}\right)\right]+(1-p) C\left[T^{\theta}\left(N^{\prime}, C_{x^{2}}\right)\right]\right]\right\} \\
& =C\left[T^{\theta}\left(N^{\prime}, C_{\bar{x}^{1}}\right)\right]+\left[p C\left[T^{\theta}\left(N^{\prime} \backslash\{m\}, C_{\bar{x}^{2} \backslash\{m\}}\right)\right]+(1-p) C\left[T^{\theta}\left(N^{\prime}, C_{\bar{x}^{2}}\right)\right]\right],
\end{aligned}
$$

where $p=\frac{\sum_{(i, j) \in B^{m}} c_{i j}}{C\left[T^{\theta}\left(N^{\prime}, C_{x}\right)\right]}$. The strategies $\bar{x}_{i}(\cdot), i \in N$, are said to be cooperative, and $\bar{x}(\cdot)=\left(\bar{x}_{1}, \ldots, \bar{x}_{n}\right)$ is called a cooperative strategy profile. In addition, $C\left[T^{\theta}\left(N^{\prime}, C_{x}\right)\right]$ and $C\left[T^{\theta}\left(N^{\prime} \backslash\{m\}, C_{x \backslash\{m\}}\right)\right]$ denote the total cost on the edges in the perishable goods trees $T^{\theta}\left(N^{\prime}, C_{x}\right)$ and $T^{\theta}\left(N^{\prime} \backslash\{m\}, C_{x \backslash\{m\}}\right)$, respectively (see Definition 22).

Definition 24. Define the characteristic function for the coalition $S \varsubsetneqq N, S^{\prime}=$ $S \cup\{0\}$, in the following way.

If $m \in S, S^{\prime}=S \cup\{0\}$, and $x_{S}(\cdot)=\left\{x_{i}(\cdot), i \in S\right\}$, then

$$
\begin{aligned}
V^{1}\left(S^{\prime}\right) & =\min _{x_{S}(\cdot)}\left\{C\left[T^{\theta}\left(S^{\prime}, C_{x_{S}^{1}}^{S^{\prime}}\right)\right]\right. \\
& \left.+\left[p C\left[T^{\theta}\left(S^{\prime} \backslash\{m\}, C_{x_{S}^{2} \backslash\{m\}}^{S^{\prime} \backslash\{m\}}\right)\right]+(1-p) C\left[T^{\theta}\left(S^{\prime}, C_{x^{2}}^{S^{\prime}}\right)\right]\right]\right\} \\
& =C\left[T^{\theta}\left(S^{\prime}, C_{\bar{x}_{S}^{1}}^{S^{\prime}}\right)\right]+\left[p C\left[T^{\theta}\left(S^{\prime} \backslash\{m\}, C_{\bar{x}_{S}^{2} \backslash\{m\}}^{S^{\prime} \backslash\{m\}}\right)\right]+(1-p) C\left[T^{\theta}\left(S^{\prime}, C_{\bar{x}_{S}^{2}}^{S^{\prime}}\right)\right]\right] .
\end{aligned}
$$

If $m \notin S, S^{\prime}=S \cup\{0\}$, and $x_{S}(\cdot)=\left\{x_{i}(\cdot), i \in S\right\}$, then

$$
\begin{aligned}
V^{1}\left(S^{\prime}\right) & =\min _{x_{S}(\cdot)}\left\{C\left[T^{\theta}\left(S^{\prime}, C_{x_{S}^{1}}^{S^{\prime}}\right)\right]+C\left[T^{\theta}\left(S^{\prime}, C_{x_{S}^{2}}^{S^{\prime}}\right)\right]\right\} \\
& =C\left[T^{\theta}\left(S^{\prime}, C_{\bar{x}_{S}^{1}}^{S^{\prime}}\right)\right]+C\left[T^{\theta}\left(S^{\prime}, C_{\bar{x}_{S}^{2}}^{S^{\prime}}\right)\right],
\end{aligned}
$$

where $p=\frac{\sum_{(i, j) \in B^{m}} c_{i j}}{C\left[T^{\theta}\left(S^{\prime}, C_{x_{S}^{1}}^{S^{\prime}}\right)\right]}$ and $C^{S^{\prime}}$ or or $C^{S^{\prime} \backslash\{m\}}$ denotes the cost matrix restricted to $S^{\prime}$ or $S^{\prime} \backslash\{m\} . x_{S}(\cdot)$ are strategy profiles of coalition $S$.

In addition, $C\left[T^{\theta}\left(S^{\prime}, C_{x_{S}}^{S^{\prime}}\right)\right]$ and $C\left[T^{\theta}\left(S^{\prime} \backslash\{m\}, C_{x_{S} \backslash\{m\}}^{S^{\prime} \backslash\{m\}}\right)\right]$ denote the total cost on the edges in the perishable goods trees $T^{\theta}\left(S^{\prime}, C_{x_{S}}^{S^{\prime}}\right)$ and $T^{\theta}\left(S^{\prime} \backslash\{m\}, C_{x_{S} \backslash\{m\}}^{S^{\prime} \backslash\{m\}}\right)$, respectively (see Definition 22).

If $S=\emptyset$, then

$$
V^{1}(\emptyset \cup\{0\})=0 .
$$

The characteristic function for the coalition $N$ at the second stage is defined in a similar way. 
Definition 25. If player $m$ leaves the game after the first stage, then

$$
V^{2}\left(N^{\prime} \backslash\{m\}\right)=\min _{x^{2}(\cdot)} C\left[T^{\theta}\left(N^{\prime} \backslash\{m\}, C_{x^{2} \backslash\{m\}}\right)\right]=C\left[T^{\theta}\left(N^{\prime} \backslash\{m\}, C_{\bar{x}^{2} \backslash\{m\}}\right)\right],
$$

where the strategies $\bar{x}_{i}^{2}(\cdot), i \in N \backslash\{m\}$, are said to be cooperative, and $\bar{x}^{2}(\cdot)=$ $\left(\bar{x}_{1}^{2}, \ldots, \bar{x}_{m-1}^{2}, \bar{x}_{m+1}^{2}, \ldots, \bar{x}_{n}^{2}\right)$ is called a cooperative strategy profile. In addition, $C\left[T^{\theta}\left(N^{\prime} \backslash\{m\}, C_{x^{2}} \backslash\{m\}\right)\right]$ denotes the total cost on the edges in the perishable goods tree $T^{\theta}\left(N^{\prime} \backslash\{m\}, C_{x^{2} \backslash\{m\}}\right)$ (see Definition 22).

If player $m$ does not leave the game after the first stage, then

$$
V^{2}\left(N^{\prime}\right)=\min _{x_{S}^{2}(\cdot)} C\left[T^{\theta}\left(N^{\prime}, C_{x_{S}^{2}}\right)\right]=C\left[T^{\theta}\left(N^{\prime}, C_{\bar{x}_{S}^{2}}\right)\right],
$$

where the strategies $\bar{x}_{i}^{2}(\cdot), i \in N$, are said to be cooperative, and $\bar{x}^{2}(\cdot)=\left(\bar{x}_{1}^{2}, \ldots, \bar{x}_{n}^{2}\right)$ is called a cooperative strategy profile. In addition, $C\left[T^{\theta}\left(N^{\prime}, C_{x^{2}}\right)\right]$ denotes the total cost on the edges in the perishable goods tree $T^{\theta}\left(N^{\prime}, C_{x^{2}}\right)$ (see Definition 22).

The characteristic function for the coalition $S \varsubsetneqq N, S^{\prime}=S \cup\{0\}$, at the second stage is defined in a similar way:

$$
V^{2}\left(S^{\prime}\right)=\min _{x(\cdot)} C\left[T^{\theta}\left(S^{\prime}, C_{x^{2}}^{S^{\prime}}\right)\right]=C\left[T^{\theta}\left(S^{\prime}, C_{\bar{x}^{2}}^{S^{\prime}}\right)\right]
$$

where $S \varsubsetneqq N, S^{\prime}=S \cup\{0\}$, and $C^{S^{\prime}}$ denotes the cost matrix restricted to $S^{\prime} . x_{S}(\cdot)$ are strategy profiles of coalition $S$.

In addition, $C\left[T^{\theta}\left(S^{\prime}, C_{x_{S}^{2}}^{S^{\prime}}\right)\right]$ denotes the total cost on the edges in the perishable goods tree $T^{\theta}\left(S^{\prime}, C_{x_{S}^{2}}^{S^{\prime}}\right)$ (see Definition 22).

If $S=\emptyset$, then

$$
V^{2}(\emptyset \cup\{0\})=0 .
$$

\section{Dynamic Shapley value}

Define the dynamic Shapley value, in the game itself and in each subgame.

Definition 26. The Shapley value has the form

$$
S h_{i}^{1}\left(N^{\prime}\right)=\frac{1}{n !} \sum_{\pi \in \Pi}\left[V^{1}\left(S_{\pi(i)}^{\prime} \cup\{i\}\right)-V^{1}\left(S_{\pi(i)}^{\prime}\right)\right],
$$

where $\forall i \in N, S^{\prime}=S \cup\{0\}, S \subseteq N \backslash\{i\}, N^{\prime}=N \cup\{0\}$, $\Pi$ denotes the set of all permutations on $N$, and $S_{\pi(k)}=\{i \mid \pi(i)<\pi(k)\}$.

Definition 27. If player $m$ leaves the game after the first stage, then

$$
S h_{i}^{2}\left(N^{\prime} \backslash\{m\}\right)=\frac{1}{(n-1) !} \sum_{\pi^{\prime} \in \Pi^{\prime}}\left[V^{2}\left(S_{\pi^{\prime}(i)}^{\prime} \cup\{i\}\right)-V^{2}\left(S_{\pi^{\prime}(i)}^{\prime}\right)\right],
$$

where $\forall i \in N \backslash\{m\}, S^{\prime}=S \cup\{0\}, S \subseteq N \backslash\{m, i\}, N^{\prime}=N \cup\{0\}, \Pi^{\prime}$ denotes the set of all permutations on $N \backslash\{m\}$, and $S_{\pi^{\prime}(k)}=\left\{i \mid \pi^{\prime}(i)<\pi^{\prime}(k)\right\}$.

If player $m$ stays in the game after the first stage, then

$$
S h_{i}^{2}\left(N^{\prime}\right)=\frac{1}{n !} \sum_{\pi \in \Pi}\left[V^{2}\left(S_{\pi(i)}^{\prime} \cup\{i\}\right)-V^{2}\left(S_{\pi(i)}^{\prime}\right)\right],
$$

where $\forall i \in N, S^{\prime}=S \cup\{0\}, S \subseteq N \backslash\{i\}, N^{\prime}=N \cup\{0\}$, $\Pi$ denotes the set of all permutations on $N$, and $S_{\pi(k)}=\{i \mid \pi(i)<\pi(k)\}$. 
Definition 28. The Imputation Distribution Procedure for the Shapley value in the two-stage perishable goods game is a payment scheme $\beta=\left(\beta^{1}, \beta^{2}\right)$ given by

$$
\begin{array}{r}
\beta^{1}=S h^{1}\left(N^{\prime}\right)-p S h^{2}\left(N^{\prime} \backslash\{m\}\right)-(1-p) S h^{2}\left(N^{\prime}\right), \\
\beta^{2}=p S h^{2}\left(N^{\prime} \backslash\{m\}\right)+(1-p) S h^{2}\left(N^{\prime}\right),
\end{array}
$$

where $p=\frac{\sum_{(i, j) \in B^{m}} c_{i j}}{C\left[T^{\theta}\left(N^{\prime}, C_{\bar{x} 1}\right)\right]}$ is the probability that player $m$ will leave the game at the second stage.

The dynamic Shapley values based on the Imputation Distribution Procedure (IDP) is constructed (Petrosyan, 1979).

Definition 29. The Shapley value $S h^{1}$ is said to be time-consistent in the perishable goods game if there exists a nonnegative IDP $\left(\beta_{i}^{1} \geq 0, \beta_{i}^{2} \geq 0, \forall i \in N\right)$ such that

$$
\begin{aligned}
S h^{1}\left(N^{\prime}\right)= & \beta^{1}+p S h^{2}\left(N^{\prime} \backslash\{m\}\right)+(1-p) S h^{2}\left(N^{\prime}\right), \\
& p S h^{2}\left(N^{\prime} \backslash\{m\}\right)+(1-p) S h^{2}\left(N^{\prime}\right)=\beta^{2},
\end{aligned}
$$

where $p=\frac{\sum_{(i, j) \in B^{m}} c_{i j}}{C\left[T^{\theta}\left(N^{\prime}, C_{\bar{x}^{1}}\right)\right]}$ is the probability that player $m$ will leave the game at the second stage.

Unfortunately, the IDP $\beta$ may have a negative values in the two-stage perishable goods game.

Proposition 1. The IDP $\beta$ for the dynamic Shapley value $S h^{1}=\left(S h_{1}^{1}, \ldots, S h_{n}^{1}\right)$ constructed by Definition 28 is time-inconsistent.

For proving this result, we provide an example when the IDP for the dynamic Shapley value is time-inconsistent.

Example 5. Consider a two-stage perishable goods game of two players as follows. $N=\{1,2\},\{0\}$ is the source, and $N^{\prime}=N \cup\{0\}$. Assume that player 2 may leave the game after the first stage.

This game is shown in Fig. 7 .

The cost on the edges $(0,1),(0,2)$, the cost of perishable goods and the loss parameters are shown in Table 2.

Table 2. The cost on the edges $(0,1),(0,2)$ and other parameters for perishable goods

\begin{tabular}{|c|c|c|c|c|c|c|c|}
\hline$c_{01}$ & $c_{10}$ & $c_{02}$ & & \multicolumn{2}{|c|}{ Player 1 } & \multicolumn{2}{|c|}{ Player 2 } \\
\cline { 4 - 7 } & & & $q_{1}$ & $\theta_{1}$ & $q_{2}$ & $\theta_{2}$ \\
\hline 80 & 80 & 10 & 10 & 4 & 10 & 2 & 15 \\
\hline
\end{tabular}

Suppose that the sets of actions of player 1(2) against player 2(1) are

$$
X_{1,2}=\{2,4\}, X_{2,1}=\{3,5\} .
$$

The function $f_{c}$ has the form $f_{c}=x_{1,2} x_{2,1}$, where $x_{1,2} \in X_{1,2}$ and $x_{2,1} \in X_{2,1}$. 


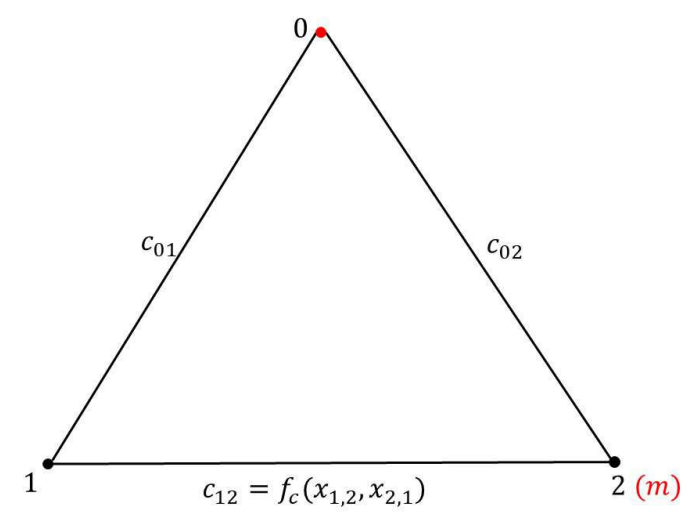

Fig. 7. Two-stage perishable goods game of two players.

For this two-stage game, we obtain the following cooperative strategy of the player 1: $\bar{x}_{1}=\left(\bar{x}_{1}^{1}, \bar{x}_{1}^{2}\right)=(2,2)$. And cooperative strategy of the player 2 : $\bar{x}_{2}=$ $\left(\bar{x}_{2}^{1}, \bar{x}_{2}^{2}\right)=(3,3)$.

In two-stage games and the subgame, the characteristic functions $S \varsubsetneqq N$ and the Shapley values of the players are shown in Table 3 .

Table 3. The characteristic functions $S \varsubsetneqq N$ and the Shapley values of players $(p=0.375)$.

\begin{tabular}{|c|c|c|c|c|}
\hline \multicolumn{5}{|c|}{ Stage 1 } \\
\hline$V^{1}\left(N^{\prime}\right)$ & $V^{1}(\{1\} \cup\{0\})$ & $V^{1}(\{2\} \cup\{0\})$ & $S h_{1}^{1}\left(N^{\prime}\right)$ & $S h_{2}^{1}\left(N^{\prime}\right)$ \\
\hline 61.7219 & 160.94 & 23.1074 & 99.7772 & -38.0553 \\
\hline \multicolumn{5}{|c|}{ Stage 2(if player 2 leaves the game). } \\
\hline$V^{2}\left(N^{\prime}\right)$ & $V^{2}(\{1\} \cup\{0\})$ & $V^{2}(\{2\} \cup\{0\})$ & $S h_{1}^{2}\left(N^{\prime}\right)$ & $S h_{2}^{2}\left(N^{\prime}\right)$ \\
\hline 80.47 & 80.47 & 0 & 80.47 & 0 \\
\hline \multicolumn{5}{|c|}{ Stage 2(if player 2 stays in the game). } \\
\hline$V^{2}\left(N^{\prime}\right)$ & $V^{2}(\{1\} \cup\{0\})$ & $V^{2}(\{2\} \cup\{0\})$ & $S h_{1}^{2}\left(N^{\prime}\right)$ & $S h_{2}^{2}\left(N^{\prime}\right)$ \\
\hline 19.4127 & 80.47 & 11.5537 & 44.1645 & -24.7518 \\
\hline
\end{tabular}

As a result, we obtain the IDP

$$
\beta_{1}^{1} \approx 32.92, \beta_{2}^{1} \approx-28.77, \beta_{1}^{2} \approx 66.86, \beta_{2}^{2} \approx-9.28 .
$$

Obviously, the dynamic Shapley value in this example is time-inconsistent.

Theorem 1. In the two-stage game, there exists some player $m$ that may leave the game at the second stage with a probability depending on the choice of all players at the first stage. The dynamic Shapley value constructed by Definition 26 is time- 
consistent if, for each stage,

$$
\begin{aligned}
& p=0, \\
& \sum_{S \subseteq N \backslash\{i\}} C\left[T^{\theta}\left(S^{\prime} \cup\{i\}, C_{\bar{x}_{S}^{k}}^{S^{\prime} \cup\{i\}}\right)\right] \geq \sum_{S \subseteq N \backslash\{i\}} C\left[T^{\theta}\left(S^{\prime}, C_{\bar{x}_{S}^{k}}^{S^{\prime}}\right)\right] \\
& S^{\prime}=S \cup\{0\}, \forall i \in N, k \in\{1,2\},
\end{aligned}
$$

where $p=\frac{\sum_{(i, j) \in B^{m}} c_{i j}}{C\left[T^{\theta}\left(N^{\prime}, C_{\bar{x}^{1}}\right)\right]}$ is the probability that player $m$ will leave the game at the second stage, and $\bar{x}_{S}(\cdot)$ are strategy profiles of coalition $S$.

Proof. Using Definitions 27 and 28, we first calculate $\beta^{2}$ :

$$
\begin{aligned}
& \beta_{i}^{2}=p S h_{i}^{2}\left(N^{\prime} \backslash\{m\}\right)+(1-p) S h_{i}^{2}\left(N^{\prime}\right)=0 S h_{i}^{2}\left(N^{\prime} \backslash\{m\}\right)+(1-0) S h_{i}^{2}\left(N^{\prime}\right) \\
= & S h_{i}^{2}\left(N^{\prime}\right)=\frac{1}{n !} \sum_{\pi \in \Pi}\left[V^{2}\left(S_{\pi(i)}^{\prime} \cup\{i\}\right)-V^{2}\left(S_{\pi(i)}^{\prime}\right)\right], \forall i \in N, S \subseteq N \backslash\{i\}, S^{\prime}=S \cup\{0\} .
\end{aligned}
$$

Since $p=0$, player $m$ stays in the game at the second stage. For (9) we apply another (equivalent) formula for calculating the Shapley value (Shapley, 1952):

$$
\begin{gathered}
S h_{i}^{2}\left(N^{\prime}\right)=\frac{1}{n !} \sum_{\pi \in \Pi}\left[C\left[T^{\theta}\left(S_{\pi(i)}^{\prime} \cup\{i\}, C_{\bar{x}_{S}^{2}}^{S_{\pi(i)}^{\prime} \cup\{i\}}\right)\right]-C\left[T^{\theta}\left(S_{\pi(i)}^{\prime}, C_{\bar{x}_{S}^{2}}^{S_{\pi(i)}^{\prime}}\right)\right]\right] \\
=\sum_{S \subseteq N \backslash\{i\}} \frac{|S| !(n-|S|-1) !}{n !}\left[C\left[T^{\theta}\left(S^{\prime} \cup\{i\}, C_{\bar{x}_{S}^{2}}^{S^{\prime} \cup\{i\}}\right)\right]-C\left[T^{\theta}\left(S^{\prime}, C_{\bar{x}_{S}^{2}}^{S^{\prime}}\right)\right]\right] \\
=\frac{|S| !(n-|S|-1) !}{n !}\left[\sum_{S \subseteq N \backslash\{i\}} C\left[T^{\theta}\left(S_{\pi(i)}^{\prime} \cup\{i\}, C_{\bar{x}_{S}^{2}}^{S_{\pi(i)}^{\prime} \cup\{i\}}\right)\right]\right. \\
\left.-\sum_{S \subseteq N \backslash\{i\}} C\left[T^{\theta}\left(S^{\prime}, C_{\bar{x}_{S}^{2}}^{S^{\prime}}\right)\right]\right], \forall i \in N, S^{\prime}=S \cup\{0\} .
\end{gathered}
$$

From conditions (10) we obtain

$$
\begin{aligned}
& \sum_{S \subseteq N \backslash\{i\}} C\left[T^{\theta}\left(S^{\prime} \cup\{i\}, C_{\bar{x}_{S}^{2}}^{S^{\prime} \cup\{i\}}\right)\right] \geq \sum_{S \subseteq N \backslash\{i\}} C\left[T^{\theta}\left(S^{\prime}, C_{\bar{x}_{S}^{2}}^{S^{\prime}}\right)\right] \\
& \beta_{i}^{2}=S h_{i}^{2}\left(N^{\prime}\right)=\frac{1}{n !} \sum_{\pi \in \Pi}\left[V^{2}\left(S_{\pi(i)}^{\prime} \cup\{i\}\right)-V^{2}\left(S_{\pi(i)}^{\prime}\right)\right] \geq 0,
\end{aligned}
$$

where $\forall i \in N, S \subseteq N \backslash\{i\}, S^{\prime}=S \cup\{0\}, \Pi$ denotes the set of all permutations on $N, S_{\pi(k)}=\{i \mid \pi(i)<\pi(k)\}$, and $\bar{x}_{S}(\cdot)=\left(\bar{x}_{i}, i \in S\right)$ are strategy profiles of coalition $S$.

Next, we consider $\beta^{1}, \forall i \in N$ :

$$
\begin{gathered}
\beta_{i}^{1}=S h_{i}^{1}\left(N^{\prime}\right)-p S h_{i}^{2}\left(N^{\prime} \backslash\{m\}\right)-(1-p) S h_{i}^{2}\left(N^{\prime}\right) \\
=S h_{i}^{1}\left(N^{\prime}\right)-0 p S h_{i}^{2}\left(N^{\prime} \backslash\{m\}\right)-(1-0) S h_{i}^{2}\left(N^{\prime}\right)=S h_{i}^{1}\left(N^{\prime}\right)-S h_{i}^{2}\left(N^{\prime}\right) .
\end{gathered}
$$

Using the equivalent formula for the Shapley value together with Definitions 26 and 27 , we find:

$$
S h_{i}^{1}\left(N^{\prime}\right)-S h_{i}^{2}\left(N^{\prime}\right)
$$




$$
\begin{gathered}
=\frac{1}{n !} \sum_{\pi \in \Pi}\left[V^{1}\left(S_{\pi(i)}^{\prime} \cup\{i\}\right)-V^{1}\left(S_{\pi(i)}^{\prime}\right)\right]-\frac{1}{n !} \sum_{\pi \in \Pi}\left[V^{2}\left(S_{\pi(i)}^{\prime} \cup\{i\}\right)-V^{2}\left(S_{\pi(i)}^{\prime}\right)\right] \\
=\sum_{S \subseteq N \backslash\{i\}} \frac{|S| !(n-|S|-1) !}{n !}\left[V^{1}\left(S^{\prime} \cup\{i\}\right)-V^{1}\left(S^{\prime}\right)\right] \\
\quad-\sum_{S \subseteq N \backslash\{i\}} \frac{|S| !(n-|S|-1) !}{n !}\left[V^{2}\left(S^{\prime} \cup\{i\}\right)-V^{2}\left(S^{\prime}\right)\right] \\
=\sum_{S \subseteq N \backslash\{i\}} \frac{|S| !(n-|S|-1) !}{n !}\left[V^{1}\left(S^{\prime} \cup\{i\}\right)-V^{1}\left(S^{\prime}\right)-V^{2}\left(S^{\prime} \cup\{i\}\right)+V^{2}\left(S^{\prime}\right)\right],
\end{gathered}
$$

where $i \in N, S^{\prime}=S \cup\{0\}, S \subseteq N \backslash\{i\}, N^{\prime}=N \cup\{0\}$, $\Pi$ denotes the set of all permutations on $N$, and $S_{\pi(k)}=\{i \mid \pi(i)<\pi(k)\}$.

We utilize the characteristic function defined as described before. For $S \varsubsetneqq N$, if $m \in S, S^{\prime}=S \cup\{0\}$, then

$$
\begin{gathered}
V^{1}\left(S^{\prime}\right)=C\left[T^{\theta}\left(S^{\prime}, C_{\bar{x}_{S}^{1}}^{S^{\prime}}\right)\right]+\left[p C\left[T^{\theta}\left(S^{\prime} \backslash\{m\}, C_{\bar{x}_{S}^{2} \backslash\{m\}}^{S^{\prime} \backslash\{m\}}\right)\right]+(1-p) C\left[T^{\theta}\left(S^{\prime}, C_{\bar{x}_{S}^{2}}^{S^{\prime}}\right)\right]\right] \\
=C\left[T^{\theta}\left(S^{\prime}, C_{\bar{x}_{S}^{1}}^{S^{\prime}}\right)\right]+\left[0 C\left[T^{\theta}\left(S^{\prime} \backslash\{m\}, C_{\bar{x}_{S}^{2} \backslash\{m\}}^{S^{\prime} \backslash\{m\}}\right)\right]+(1-0) C\left[T^{\theta}\left(S^{\prime}, C_{\bar{x}_{S}^{2}}^{S^{\prime}}\right)\right]\right] \\
=C\left[T^{\theta}\left(S^{\prime}, C_{\bar{x}_{S}^{1}}^{S^{\prime}}\right)\right]+C\left[T^{\theta}\left(S^{\prime}, C_{\bar{x}_{S}^{2}}^{S^{\prime}}\right)\right] .
\end{gathered}
$$

If $m \notin S, S^{\prime}=S \cup\{0\}, x_{S}(\cdot)=\left\{x_{i}(\cdot), i \in S\right\}$, then

$$
\begin{aligned}
V^{1}\left(S^{\prime}\right) & =\min _{x_{S}(\cdot)}\left[C\left[T^{\theta}\left(S^{\prime}, C_{x_{S}^{1}}^{S^{\prime}}\right)\right]+C\left[T^{\theta}\left(S^{\prime}, C_{x_{S}^{2}}^{S^{\prime}}\right)\right]\right] \\
= & C\left[T^{\theta}\left(S^{\prime}, C_{\bar{x}_{S}^{\prime}}^{S^{\prime}}\right)\right]+C\left[T^{\theta}\left(S^{\prime}, C_{\bar{x}_{S}^{2}}^{S^{\prime}}\right)\right] .
\end{aligned}
$$

Since $p=0$, player $m$ stays in the game at the second stage. Therefore, formulas (8), (9), (12),(13) and (14) imply

$$
\begin{aligned}
V^{1}\left(S^{\prime}\right)-V^{2}\left(S^{\prime}\right) & =C\left[T^{\theta}\left(S^{\prime}, C_{\bar{x}_{S}^{1}}^{S^{\prime}}\right)\right]+C\left[T^{\theta}\left(S^{\prime}, C_{\bar{x}_{S}^{2}}^{S^{\prime}}\right)\right]-C\left[T^{\theta}\left(S^{\prime}, C_{\bar{x}_{S}^{2}}^{S^{\prime}}\right)\right] \\
& =C\left[T^{\theta}\left(S^{\prime}, C_{\bar{x}_{S}^{1}}^{S^{\prime}}\right)\right], S \varsubsetneqq N,
\end{aligned}
$$

where $\bar{x}_{S}(\cdot)=\left(\bar{x}_{i}, i \in S\right)$ are strategy profiles of coalition $S$.

Thus, from (12) and (15) we obtain

$$
\begin{gathered}
S h_{i}^{1}\left(N^{\prime}\right)-S h_{i}^{2}\left(N^{\prime}\right) \\
=\sum_{S \subseteq N \backslash\{i\}} \frac{|S| !(n-|S|-1) !}{n !}\left[\left(V^{1}\left(S^{\prime} \cup\{i\}\right)-V^{2}\left(S^{\prime} \cup\{i\}\right)\right)\right. \\
\left.-\left(V^{1}\left(S^{\prime}\right)-V^{2}\left(S^{\prime}\right)\right)\right]=\sum_{S \subseteq N \backslash\{i\}} \frac{|S| !(n-|S|-1) !}{n !}\left[C\left[T^{\theta}\left(S^{\prime} \cup\{i\}, C_{\bar{x}_{S}^{1}}^{S^{\prime} \cup\{i\}}\right)\right]\right. \\
\left.-C\left[T^{\theta}\left(S^{\prime}, C_{\bar{x}_{S}^{1}}^{S^{\prime}}\right)\right]\right]=\frac{|S| !(n-|S|-1) !}{n !}\left[\sum_{S \subseteq N \backslash\{i\}} C\left[T^{\theta}\left(S^{\prime} \cup\{i\}, C_{\bar{x}_{S}^{1}}^{S^{\prime} \cup\{i\}}\right)\right]\right. \\
\left.-\sum_{S \subseteq N \backslash\{i\}} C\left[T^{\theta}\left(S^{\prime}, C_{\bar{x}_{S}^{1}}^{S^{\prime}}\right)\right]\right], \forall i \in N
\end{gathered}
$$


From (10), (11) and (16) we obtain:

$$
\begin{gathered}
\sum_{S \subseteq N \backslash\{i\}} C\left[T^{\theta}\left(S^{\prime} \cup\{i\}, C_{\bar{x}_{S}^{1}}^{S^{\prime} \cup\{i\}}\right)\right] \geq \sum_{S \subseteq N \backslash\{i\}} C\left[T^{\theta}\left(S^{\prime}, C_{\bar{x}_{S}^{1}}^{S^{\prime}}\right)\right] \\
\beta_{i}^{1}=S h_{i}^{1}\left(N^{\prime}\right)-S h_{i}^{2}\left(N^{\prime}\right) \geq 0,
\end{gathered}
$$

where $\forall i \in N, S \subseteq N \backslash\{i\}, S^{\prime}=S \cup\{0\}, \Pi$ denotes the set of all permutations on $N, S_{\pi(k)}=\{i \mid \pi(i)<\pi(k)\}$, and $\bar{x}_{S}(\cdot)=\left(\bar{x}_{i}, i \in S\right)$ are strategy profiles of coalition $S$.

The proof of Theorem 1 is complete.

\section{Conclusion}

In this paper, we study two-stage minimum spanning tree games with perishable goods. A particular player leaves the game at the end of the first stage with a certain probability. This probability depends on the behaviour of all players in the first stage. A dynamic Shapley value is constructed by defining characteristic functions of the cooperative game. Moreover, a theorem is presented in which the dynamic Shapley value is time consistent if specific conditions are satisfied.

\section{References}

Bergantiños, G. and Vidal-Puga, J. J. (2007). A fair rule in minimum cost spanning tree problems. Journal of Economic Theory, 137(1), 326-352.

Bird, C.G. (1976). On cost allocation for a spanning tree: A game theoretic approach. Networks, 6(4), 335-350.

Claus, A. and Kleitman, D. J. (1973). Cost allocation for a spanning tree. Networks. Wiley Online Library, 3(4), 289-304.

Dijkstra, E. W. (1959). A note on two problems in connexion with graphs. Numerische Mathematik, 1(1), 269-271.

Dutta, B. and Kar, A. (2004). Cost monotonicity, consistency and minimum cost spanning tree games. Games and Economic Behavior, 48(2), 223-248.

Janssen, L., Claus, T. and Sauer, J. (2016). Literature review of deteriorating inventory models by key topics from 2012 to 2015. International Journal of Production Economics. Elsevier, 182, 86-112.

Kruskal, J. B. (1956). On the shortest spanning subtree of a graph and the traveling salesman problem. Proceedings of the American Mathematical society, 7(1), 48-50.

Nahmias, S. (1982). Perishable Inventory Theory: a Review. Operations Research, 30(4), 680-708.

Prim, R. C. (1957). Shortest Connection Networks And Some Generalizations. Bell System Technical Journal, 36(6), 1389-1401.

Wan, G., Li, Q. and Cao, Y. (2019). Optimal price markdown strategy for perishable food products considering retailers service level. The theory and practice of finance and economics, 40(5), 142-147 (in Chinese).

Li, Y. (2016). The dynamic Shapley Value in the game with spanning tree. In Proceedings of 2016 International Conference "Stability and Oscillations of Nonlinear Control Systems" (Pyatnitskiy's Conference), STAB 2016.

Li, Y. (2017). Dynamic Shapley Value for 2-stage cost sharing game with perishable products. In Proceedings of the 29th Chinese Control and Decision Conference, CCDC 2017, 3770-3774.

Petrosyan, L. A., and Danilov, N. N. (1979). Stability of solutions in non-zero sum differential games with transferable payoffs. Viestnik of Leningrad Universtiy, 1, 52-59.

Shapley, L.S. (1952). A value for $n$-person games. Rand corp santa monica ca. 